\title{
Musculoskeletal disorders in patients with hyperlipidaemia
}

\author{
G. R. STRUTHERS, D. L. SCOTT, P. A. B ACON, AND K. W. WALTON \\ From the Rheumatism Research Wing, The Medical School, University of Birmingham, Birmingham B15 2TJ
}

SUMMARY We have assessed the nature and significance of musculoskeletal disorders in 166 patients with the commonly encountered forms of hyperlipidaemia attending a lipid clinic. The incidence of musculoskeletal disorders was determined by questionnaire to the patients, inquiries to general practitioners, and a review of hospital records. To ensure the sensitivity of these methods a series of negatively responding cases were seen and examined; none had identifiable musculoskeletal disease. Eight males with type IV hyperlipidaemia had recurrent acute attacks of gout. The only other musculoskeletal disorder possibly attributable to an association with hyperlipidaemia was a transient polyarthritis in 3 patients. This was inflammatory, recurrent, but nondeforming, and involved the small joints of the hand. It did not require any specific therapy or lead to disability. We suggest that, while we have confirmed the association of type IV hyperlipidaemia and gout, there is little evidence for clinically significant arthritis being associated with other commonly presenting forms of hyperlipidaemia, although we accept that the rarely met homozygous familial hypercholesterolaemia may also be complicated by a crystal arthropathy due to the presence of crystalline cholesterol in joints.

Hyperlipidaemia can be familial or acquired. ${ }^{1}$ In addition to its well documented role in the formation of atheroma ${ }^{2}$ it has been associated with a variety of musculoskeletal disorders. ${ }^{3}$ The best known association is of hypertriglyceridaemia with gout. ${ }^{4}$ But other reported associations include a migratory polyarthritis with familial hypercholesterolaemia ${ }^{56}$ and acute tendinitis ${ }^{7}$ and various nonspecific musculoskeletal symptoms with less well defined hyperlipidaemias. ${ }^{89}$ However, the reported associations have been observed in highly selected groups of patients, and their significance in relation to the whole spectrum of lipid and lipoprotein abnormalities is unclear. A recent survey in Sweden has suggested that there is little correlation between lipid/lipoprotein levels and musculoskeletal disease, ${ }^{10}$ and this has led us to evaluate further this relationship. We now report the incidence, nature, and clinical significance of musculoskeletal disease in patients attending a lipid clinic in Birmingham.

\section{Patients and methods}

Patients entered in study. All patients who were on the

Accepted for publication 4 October 1982.

Correspondence to Dr G. R. Struthers, Rheumatism Research Wing, Medical School, University of Birmingham, Birmingham B15 2TJ. register of the lipid clinic held in the Department of Investigative Pathology between 1974 and 1980 were included. There were 231 patients in the clinic register (M/F: 154/77).

Questionnaires. Both patients and their general practitioners were sent initial questionnaires (Tables 1 and 2) based on one designed by Cobb. ${ }^{11}$ They were sent to all the patients entered in the clinic register. Those who did not reply were sent a second questionnaire, and if this failed to elicit a response were contacted by telephone if this was possible.

Lipoprotein analysis. The hyperlipidaemias encountered were classified as suggested by Beaumont et al. ${ }^{12}$ Lipoprotein estimations were performed

\section{Table 1 Patients' questionnaire \\ 1 Have you at any time had arthritis or rheumatism? \\ 2 Do you wake up with stiffness or aching in your joints or muscles? \\ 3 Have you ever had swelling of any joints?}

Table 2 General practitioners' questionnaire

\begin{tabular}{ll}
\hline Has your patient: \\
1 Ever consulted you with arthritis or rheumatism? \\
2 Ever complained of stiffness or aching in the joints and muscles? \\
3 Ever had swelling of any joints?
\end{tabular}

Ever consulted you with arthritis or rheumatism?

3 Ever had swelling of any joints? 
in serum specimens taken after an overnight fast of .nore than 9 hours. The estimations were performed at the Wolfson Research Laboratories of the University of Birmingham by standard automated techniques. ${ }^{13}$ The first available lipoprotein analysis in the records of the lipid clinic was used for classification.

Patients excluded. Sixty-five patients were excluded from the study for the reasons shown in Table 3. Since 27 patients had normal lipids and 4 had never attended, only 33 patients were strictly speaking lost to the study. Of these, $10 \mathrm{died}$. This left 166 patients (M/F: 116:50) who were all included in the study.

Clinic visit. All patients who replied in the affirmative to one or more of the questions were invited to attend the clinic (Table 4). There a full history was elicited, a physical examination undertaken, and appropriate investigations performed. These investigations included full blood count, ESR; and $C$-reactive protein measurement, Rose-Waaler titre and latex test for rheumatoid factor, antinuclear factor, biochemical profile, serum uric acid, and radiographs of any involved joints.

Home visit. Patients who were unwilling or unable to attend the clinic were all seen at home. Similar assessments were made and blood tests taken if necessary. Radiographs too were arranged if appropriate and at the patient's convenience.

General practitioners' assessment. $82.6 \%$ of general practitioners replied to the questionnaires and in the majority of cases either confirmed our clinical findings or had no records of the patients' complaints. In these latter instances the patients'

Table 3 Patients excluded from study

\begin{tabular}{lrrr}
\hline Reasons for exclusion & $M$ & \multicolumn{1}{c}{$F$} & Total \\
\hline Deceased & 5 & 5 & 10 \\
Normal lipids at first attendance & 14 & 13 & 27 \\
Lost to follow-up & 15 & 8 & 23 \\
Never attended lipid clinic & 4 & 1 & 5 \\
Total & 38 & 27 & 65 \\
\hline
\end{tabular}

Table 4 Patients in study

\begin{tabular}{|c|c|c|c|c|}
\hline \multirow[t]{2}{*}{$\begin{array}{l}\text { Type of } \\
\text { hyperlipidaemia }\end{array}$} & \multicolumn{2}{|c|}{$\begin{array}{l}\text { Positive responders } \\
\text { to questionnaire }\end{array}$} & \multicolumn{2}{|c|}{$\begin{array}{l}\text { Negative responders } \\
\text { to questionnaire }\end{array}$} \\
\hline & $\boldsymbol{M}$ & $F$ & $\boldsymbol{M}$ & $\boldsymbol{F}$ \\
\hline $\begin{array}{l}\text { I/V } \\
\text { II } \\
\text { III } \\
\text { IV }\end{array}$ & $\begin{array}{r}1 \\
23 \\
3 \\
34\end{array}$ & $\begin{array}{r}0 \\
16 \\
1 \\
17\end{array}$ & $\begin{array}{r}7 \\
16 \\
1 \\
30\end{array}$ & $\begin{array}{l}2 \\
9 \\
2 \\
4\end{array}$ \\
\hline $\begin{array}{l}\text { Total } \\
\text { Mean age }\end{array}$ & $\begin{array}{l}61 \\
45 \cdot 5\end{array}$ & $\begin{array}{l}34 \\
56 \cdot 3\end{array}$ & $\begin{array}{l}54 \\
48 \cdot 3\end{array}$ & $\begin{array}{l}17 \\
57 \cdot 6\end{array}$ \\
\hline
\end{tabular}

complaints were usually of little clinical significance. In only 9 cases when patients responded negatively to all 3 questions did the general practitioners give one or more positive reply. In 6 of these cases the complaint was of a previous self-limiting episode of low back pain-often labelled lumbago-and there was one case each of cervical spondylosis, tennis elbow, and carpal tunnel syndrome. These patients were seen and included in the analysis. One patient with type IV hyperlipidaemia was said to have gout, but he refused all attempts by us to see him.

Sensitivity of the questionnaire. To check this 25 randomly selected patients who had replied in the negative and with negative responses from their general practitioners were invited to attend the clinic. None of them had any evidence of musculoskeletal disease.

Hospital record review. All patients who had attended hospital for reasons other than hyperlipidaemia had their records examined. Any record of a previous musculoskeletal disorder was noted, and the relevant physical findings, laboratory tests, and radiographs were evaluated.

\section{Results}

The main musculoskeletal disorders and their relationships to the various Fredrickson phenotypes are summarised in Table 5. Gout was found in 8 patients with type IV hyperlipidaemia-an incidence of $12.5 \%$. Six of these patients had classical recurrent podagra with hyperuricaemia. The other 2 patients had acute synovitis of other joints (knee and ankle) in association with hyperuricaemia, and the diagnosis had been confirmed by the finding of negatively birefringent crystals to polarised light in the synovial fluid from these joints. As a group these patients showed the other features of the hyperuricaemia/hypertriglyceridaemia diathesis, with 4 patients being overweight, 3 suffering from claudication, 1 from angina, and 4 admitted to a heavy alcohol intake. There were no patients with gout in the other groups, although some patients with type II disorder had hyperuricaemia.

Three patients ( 1 male with type IV and 2 female with type II hyperlipidaemia) gave a history of transient polyarthritis. This consisted of recurrent brief episodes of swelling of the small joints of the hands, especially the proximal interphalangeal joints. The joint swelling lasted no more than 2-3 days each time and was accompanied on each occasion by minimal inflammatory features and stiffness. None of the 3 patients had sought medical advice about their condition nor had they taken any medication for it. When seen they had no clinical, laboratory, nor radiological abnormalities. 
Musculoskeletal disorders in patients with hyperlipidaemia

Table 5 Hyperlipidaemia and types of arthritis. The hyperlipidaemias are classified after Fredrickson ${ }^{12}$

\begin{tabular}{lllllrlr}
\hline $\begin{array}{l}\text { Type of } \\
\text { hyperlipidaemia }\end{array}$ & $\begin{array}{l}\text { Total no. of } \\
\text { cases }\end{array}$ & Gout & $\begin{array}{l}\text { Transient } \\
\text { polyarthritis }\end{array}$ & $\begin{array}{l}\text { Rheumatoid } \\
\text { arthritis }\end{array}$ & OA & $\begin{array}{l}\text { Periarthritis of } \\
\text { shoulder }\end{array}$ \\
\hline II & 64 & 0 & 2 & 0 & 19 & 3 & 0 \\
III & 7 & 0 & 0 & 0 & 0 & 0 & 0 \\
IV & 85 & 8 & 1 & 5 & 16 & 4 & 0 \\
I/V & $1 / 9$ & 0 & 0 & 0 & 35 & 7 & 0 \\
Total & 166 & 8 & 3 & 5 & 3 \\
\hline
\end{tabular}

*Ankylosing spondylitis (1); osteoporosis of the ankle (1); tennis elbow (1).

Seven patients ( 3 with type II and 4 with type IV hyperlipidaemia) described symptoms typical of periarthritis of the shoulder. However, clinical examination showed they formed a heterogeneous group containing cases of adhesive capsulitis, supraspinatus tendinitis, and bicipital tendinitis. None had radiological evidence of calcification in the shoulder region, and all had had mild rheumatic disease without any significant or residual disability. All had mild hyperlipidaemia.

The 35 patients (19 with type II and 16 with type IV hyperlipidaemia) with osteoarthrosis had involvement of classical sites such as the neck, back, hips, knees, and ankles. ${ }^{14}$ All patients had radiological changes to support the clinical diagnosis. There were 5 patients with rheumatoid arthritis (all with type IV hyperlipidaemia). All had classical disease by the criteria of the American Rheumatism Association. ${ }^{15}$

A further 3 patients with type IV hyperlipidaemia had other conditions - one each of ankylosing spondylitis, tennis elbow, and osteoporosis of the ankle.

Finally, there were 34 patients who gave affirmative replies to at least one question in the questionnaire but who did not have definite musculoskeletal disease. Most of these had occasional aches and pains of little or no clinical significance; others had symptoms in association with other disorders, for instance, intermittent claudication, previous Pott's fracture of the ankle, or a congenital abnormality such as club foot. Some patients had given inappropriate responses.

We examined the records of all the patients with musculoskeletal disease to determine how many had been referred to the lipid clinic after presenting to another clinic with an arthritic condition. Four of the patients with gout and 3 with rheumatoid arthritis had been referred in this manner. There was no other evidence of a possible bias.

\section{Discussion}

Our study confirms several previous observa- tions $^{4}{ }^{16-20}$ of a significant association between clinical gout and hypertriglyceridaemia (type IV hyperlipidaemia). This association is not necessarily causal. Instead it may arise because of the concurrence in some patients of the gouty diathesis with precipitating factors (such as a tendency to indulge in dietary indiscretions, obesity, and increased alcohol intake) which are inherent in the life-style of these individuals. Indirect support for this proposal was provided by the observations of Darlington et al. ${ }^{21}$ who found that the first-degree relatives of gout patients did not show the hypertriglyceridaemias found in the index patients.

Raised triglyceride levels have been reported in between 52 and $84 \%^{16}{ }^{17}{ }^{22}{ }^{23}$ of patients with clinical gout but were not found in individuals with symptomless hyperuricaemia. ${ }^{16}$ When the association is sought from the converse end, as in the present study, we observed that 77 out of 85 of our cases of type IV hyperlipidaemia showed no evidence of gout. The prevalence of this type of hyperlipidaemia in hospital-derived populations is sufficiently high for us to regard it as not significant that all 5 cases of rheumatoid arthritis and almost half the cases of osteoarthrosis encountered in this study were all cases with this lipid abnormality.

There has also been speculation about an association between hypercholesterolaemia (type II hyperlipidaemia) and arthritis. Mild or moderate hyperlipidaemias of this pattern are common in most populations, but not all such patients show clear evidence of the involvement of genetic factors. In our series approximately one-third of our patients with type II hyperlipidaemia were judged (on the basis of family history and mode of presentation) to be heterozygous for familial hypercholesterolaemia. However, among this subgroup we encountered no instances of the knee effusions of the type reported by Rooney et $a l .{ }^{6}$ It was suggested recently ${ }^{24}$ that such knee effusions may also be a localised manifestation of gout demanding detailed investigation, including synovial biopsy, to make the diagnosis. Some of our cases of hypercholesterolaemia (type II hyperlipidaemia) were 
hyperuricaemic, but this was seldom severe and was unassociated with frank knee effusions. It would therefore seem that, if this subgroup, like some cases of type IV hyperlipidaemia, are indeed gouty in origin, this is a rare and unusual presentation.

In the present study we have also not seen certain other forms of musculoskeletal disorders reported by certain groups studying hyperlipidaemic patients. ${ }^{78}$ On the other hand, minor 'rheumatic' complaints, to which no definite diagnosis could be attached, were common occurring in 34 of our patients. Similar findings were reported by Goldman et al. ${ }^{9} \mathrm{~A}$ transient but recurrent synovitis of small joints of the hands was reported by 3 patients in the present study -2 with type II and 1 with type IV hyperlipidaemia (Table 5). But this was a minor disorder, resembling a mild form of palindromic rheumatism. It did not produce any deformity and did not cause the patients concerned to consult either the family doctor or a specialist. In our view it appeared therefore to be of little clinical significance.

It seems possible that the relative lack of association between musculoskeletal disorders and lipid abnormalities found in the present study derives from the fact that most of the patients surveyed were suffering from relatively mild or moderate hyperlipidaemias. There is certainly evidence from both human and animal studies that really severe hyperlipidaemias may give rise to joint disorders. For example, there can be little doubt about the reality of the association between the transient but severe arthritis reported to occur recurrently, even in young patients, and homozygous familial hypercholesterolaemia (type II familial hyperlipidaemia). According to Khachadurian ${ }^{5}$ this is a markedly disabling complication of gross hyperlipidaemia characteristic of this condition, However, this is a rare condition, no example of which was encountered among our patients.

Cholesterol has been found in joint effusions in such patients and also in other individuals with severe (presumptively heterozygous familial) hypercholesterolaemia and concurrent joint symptoms and signs. The cholesterol in such cases is thought to derive from plasma lipoproteins which gain access to inflamed joints. Crystalline cholesterol, thus derived, is thought to serve as a local irritant ${ }^{25}$ and lipid has also been found to be deposited in cartilage and intra-articular collagenous structures.

In experimental studies in rabbits, in which immune arthritis and hyperlipidaemia were induced concurrently, it was shown ${ }^{26}$ that: (i) Rabbit apolipoprotein $B$, containing lipoproteins $(\mathrm{LpB})$, which are normally virtually excluded from joint fluid by virtue of their large molecular size, gain access to inflamed joints which show altered permeability of the synovial membranes. (ii) Much of the LpB entering the joint space is taken up by phagocytic cells and hydrolysed, leaving a lipid residue. In chronically inflamed joints such residues are modified to give crystalline cholesterol and its esters which serve as tissue irritants. (iii) Intact $\mathrm{LpB}$ is sequestered in cartilage, ligaments, etc., within joints. And (iv) the severity of these changes is related to the severity of the concomitant hypercholesterolamia (hyperlipidaemia).

These observations, taken together with our own clinical study, lead us to suggest that any clinically significant joint changes occurring in association with the commonly encountered hyperlipidaemias are probably essentially crystal arthropathies. In patients presenting with the severe type II pattern of hyperlipidaemia, whether of homozygous or heterozygous familial hypercholesterolaemic origin, the offending crystal (as in experimental hypercholesterolaemic rabbits) is probably crystalline cholesterol. On the other hand in some persons with hypertriglyceridaemia (type IV hyperlipidaemia) and concurrent hyperuricaemia the crystal concerned is almost certainly urate, since the joint symptoms and signs are characteristic of classical podagra.

In contrast, in our view, any causal relation between other musculoskeletal signs and symptoms and hyperlipidaemias is unproved or of little clinical significance among the generality of mild or moderate hyperlipidaemias attending the average lipid clinic.

We thank Professor T. P. Whitehead and staff of the Wolfson Research Laboratories for carrying out the lipid profile analysis and for their kind permission to allow us to report the results here.

\section{References}

1 Fredrickson D S, Levy R I. Familial hyperlipiproteinaemia. In: Stanbury J B, Wyngaarden J B, Fredrickson D S, eds. Metabolic basis of inherited disease. New York: McGraw Hill, 1978: 604-55.

2 Mahley R W. Atherogenic hyperlipidaemia. Med Clin North Am 1982; 66: 375-402.

3 Bole G G. Arthritis associated with hyperlipidaemia and hypercholesterolaemia. In: Kelley W N, Harris E D, Ruddy S, Sledge C B, eds. Textbook of rheumatology. Philadelphia: Saunders, 1982: 1638-45.

4 Gibson T, Grahame R. Gout and hyperlipidaemia. Ann Rheum Dis 1974; 35: 298-303.

5 Khachadurian A K. Migratory polyarthritis in familial hypercholesterolaemia (type II hyperlipoproteinaemia) Arthritis Rheum 1968; 11: 385-93.

6 Rooney P J, Madkour M M, Spencer D, Carson Dick W. Transient polyarthritis associated with familial hyperlipoproteinaemia. Q J Med 1978; 47: 249-59.

7 Glueck C J, Levy R I, Fredrickson D S. Acute tendinitis and arthritis: a presenting symptom of familial type II hyperlipoproteinaemia. JAMA 1968; 13: 2895-7.

8 Buckingham R, Bole G, Bassett D. Polyarthritis associated with type IV hyperlipoproteinaemia. Arch Intern Med 1975; 135: 286-90.

9 Goldman J A, Glueck C J, Abrams N R, Steiner P, Herman J H. 
Musculo-skeletal disorders associated with type IV hyperlipidaemia. Lancet 1972; ii: 449-52.

10 Weilin L, Larson B, Svarsudd K, Tibbin G. Serum lipids, lipoproteins and musculoskeletal disorders among 50 and 60 year old men. Scand J Rheumatol 1978; 7: 7-12.

11 Cobb S. Genetics and the epidemiology of chronic diseases. US Department of Health Education and Welfare, Public Health Services, 1965: 209.

12 Beaumont J L, Carlson L A, Cooper G R, Fejfar Z, Fredrickson D S, Stasser T. Classification of hyperlipidaemias and hyperlipoproteinaemias. Bull WHO 1970; 43: 891 -908.

13 Bradby G V H, Valente A J, Walton K W. Serum high density lipoproteins in peripheral vascular disease. Lancet 1978; ii: 1271-4.

14 Lawrence J S. Rheumatism in populations. London: Heinemann, 1977.

15 Ropes M W, Bennett E A, Cobb S, Joux R and Jessar R. Revision of diagnostic criteria for rheumatoid arthritis. Bull Rheum Dis 1958; 9: 195.

16 Benedek T G. Correlations of serum uric acid and lipid concentrations in normal, gouty and atherosclerotic men. Ann Intern Med 1967; 66: 851-61.
17 Barlow K A. Hyperlipidaemia in primary gout. Metabolism 1968; 17: 289-99.

18 Feldman E B, Wallace S L. Hypertriglyceridaemia in gout. Circulation 1964; 29: 508-13.

19 Darlington L G, Scott J T. Plasma lipid levels in gout. Ann Rheum Dis 1972; 31: 487-9.

20 Frank O. Observations concerning the incidence of lipid and carbohydrate metabolism in gout. Ann Med Exp Biol Fenn 1974; 41: 495-8.

21 Darlington L G, Gleuck J, Scott J T. Family study of lipid and purine levels in gout patients. Ann Rheum Dis 1982; 41: 253-6.

22 Berkowitz D. Blood lipid and uric acid interrelationships. JAMA 1964; 190: 856-8.

23 Berkowitz D. Gout hyperlipidaemia and diabetes interrelationships. JAMA 1966; 197: 117-20.

24 Zoppini A, Teodari S, Taecari E. Valeur de la biopsie synoviale dans le diagnostic des arthropathies associées aux hyperlipoproteinaemias. Rev Rhum Mal Osteoartic 1980; 47: 111-5.

25 Zucker J, Uddin J, Gantner G E, Dorner R W. Cholesterol crystals in synovial fluid. Ann Intern Med 1964; 60: 436-46.

26 Valente A J, Walton K W. Studies on increased vascular permeability in the pathogenesis of lesions of connective tissue diseases: 1. Experimental hyperlipidaemia and immune arthropathy. Ann Rheum Dis 1980; 37: 490-9.

\section{Book review}

American Academy of Orthopaedic Surgeons: Symposium on Heritable Disorders of Connective Tissue. Edited by W. H. Akeson. P. Bornstein, M. J. Glimcher. Pp. 380. £43.50. C. V. Mosby: London. 1982.

This book reports a symposium on heritable disorders of connective tissue held in San Diego in 1980 under the auspices of the American Academy of Orthopaedic Surgeons. Twelve of the 30 chapters describe various aspects of Ehlers-Danlos syndrome, Marfan's syndrome, and osteogenesis imperfecta. Logically these are preceded by several chapters on the molecular biology of collagen, the first of which provides a glimpse into the fascinating world of the techniques of recombinant DNA which are already being used to study collagen gene structure. The last 7 chapters are about the difficult and confusing area of the chondro-osseous dysplasias - their classification, morphology, and orthopaedic management. Slotted in between are excellent chapters on the distinctly tangential subjects of bone phosphoprotein, osteocalcin and the collagen in the contractures of Dupuytren's fasciitis and immobilised joints.

The book comes from the same publisher as McCusick's classic on Heritable Disorders of Connective Tissue, the last edition of which came out in 1972; and the two are alike in quality and style. If not a mini-McCusick, the symposium is a useful up-date, which at $£ 42$ will doubtless grace the libraries of rheumatic, orthopaedic, and genetic units.

J. BALL 